\title{
Performance of blind and sighted humans on a tactile grating detection task
}

\author{
DANIEL GOLDREICH and INGRID M. KANICS \\ Duquesne University, Pittsburgh, Pennsylvania
}

\begin{abstract}
We compared the abilities of blind and sighted humans to distinguish grooved from smooth surfaces pressed against the stationary index fingertip. Ranging in age from 20 to 72 years, 37 blind and 47 sighted subjects participated in an automated two-alternative forced-choice tactile grating detection task. The tactile acuity of blind and sighted subjects declined with age at equivalent rates $(0.011-\mathrm{mm}$ threshold increase per year), but the blind subjects were able to perceive significantly thinner grooves than were their sighted peers (the average difference between blind and sighted subjects of the same age and gender was $0.267 \mathrm{~mm}$ ). The blind Braille readers performed no better than the blind nonreaders, and the congenitally blind subjects performed equivalently to those with adult-onset blindness. The superior tactile acuity of blind persons may result from the involvement of normally visually responsive cerebrocortical areas in tactile processing, as shown by functional-imaging studies.
\end{abstract}

Areas of the cerebral cortex deprived of their normal sensory inputs undergo dramatic functional changes. In blind persons, normally visual cortical areas acquire tactile (see Gizewski, Gasser, de Greiff, Boehm, \& Forsting, 2003; Sadato et al., 1998) and auditory (see Gougoux, Zatorre, Lassonde, Voss, \& Lepore, 2005; Weeks et al., 2000) responsiveness, and in deaf persons, normally auditory cortical regions are activated by vision (see Finney, Clementz, Hickok, \& Dobkins, 2003) and touch (see Levänen, Jousmaki, \& Hari, 1998).

The perceptual consequence of this cortical plasticity is unclear and is the subject of active research. Of particular interest is whether the recruitment of occipital cortex in blind persons results in enhanced acuity in the nonvisual senses. Many early studies failed to find evidence of such sensory enhancement (for reviews, see Axelrod, 1959; Hollins, 1989; Warren, 1978). However, several recent studies using modern psychophysical methods have found enhanced tactile (see Goldreich \& Kanics, 2003; Stevens, Foulke, \& Patterson, 1996; Van Boven, Hamilton, Kauffman, Keenan, \& Pascual-Leone, 2000) and auditory (see Hugdahl et al., 2004; Lessard, Paré, Lepore, \& Lassonde, 1998; Röder et al., 1999) discrimination abilities in blind persons.

A previous study from our laboratory (Goldreich \& Kanics, 2003) found that blind subjects outperformed the sighted in their ability to discriminate the orientations of thinly grooved surfaces (tactile gratings) pressed into the stationary index fingertip. The orientation of a tactile grating is thought to be represented by the locations of

The authors thank T. Chadha for assistance with experiments and D. Gillespie for comments on the manuscript. This research was supported by National Eye Institute Grant R15 EY013649-01. Address all correspondence concerning this article to D. Goldreich, Department of Psychology, Neuroscience \& Behaviour, McMaster University, Hamilton, ON, L8S 4K1 Canada (e-mail: goldrd@mcmaster.ca). activated slowly adapting Type I (SAI) afferents (see, e.g., Gibson \& Craig, 2002; Johnson \& Phillips, 1981; Phillips \& Johnson, 1981), corresponding to the positions of the edges in the grating. The superior performance of blind persons on this task therefore suggests that they more accurately decode the spatial structure of the activated SAI afferent array through central processing mechanisms.

Having found differences in performance between blind and sighted subjects on grating orientation discrimination, we next asked whether these differences would persist in a grating detection task in which subjects attempted to distinguish a thinly grooved surface from a smooth one. Because SAI afferents respond well to edges, grooved surfaces evoke greater overall SAI population response magnitudes than do smooth surfaces (Johnson \& Phillips, 1981; Phillips \& Johnson, 1981). Thus, unlike grating orientation discrimination, grating detection need not rely on the activated SAI spatial structure cue. We were interested in determining whether blind subjects would make better use than sighted subjects of the SAI population response magnitude cue present in the grating detection task.

We report here that, as was the case with grating orientation discrimination (Goldreich \& Kanics, 2003), blind subjects significantly outperformed the sighted at grating detection. Furthermore, blind subjects' tactile acuity depended neither upon the age at which vision was lost nor upon Braille reading experience. These results confirm that tactile acuity is enhanced in blindness and provide clues to the cause.

\section{METHOD}

In the experiments described, 47 sighted and 37 profoundly blind subjects participated. The subjects were screened to exclude those with diabetes, peripheral neuropathies, nervous system disorders, learning disorders, dyslexia, and injuries to or calluses on their tested index fingers. The sighted-subject group consisted of 24 women and 
23 men ranging in age from 20 years 7 months to 71 years 7 months (median 44 years 2 months); the blind group consisted of 18 women and 19 men ranging in age from 19 years 8 months to 71 years (median 49 years). All the subjects had previously participated in the study described in Goldreich and Kanics (2003). All procedures were approved by Duquesne University's Institutional Review Board for the protection of human subjects.

The blind group was heterogeneous with respect to three factors of interest: degree of light perception, Braille reading, and childhood vision. At the time of testing, 19 subjects had no light perception, and 18 had residual light perception (the ability to distinguish light from dark, and, in some cases, to discern vague shapes). Whereas 29 subjects read Braille, 8 did not. Childhood vision was classified as normal in 12 subjects (fully sighted until at least 12 years of age), low in 6 (visually impaired at birth, but able to read print with magnification throughout childhood), residual in 10 (either born with residual light perception or progressed to residual light perception or less during childhood), and none in 9 (born without light perception or lost all light perception within the first few months of life).

We assessed the ability of the subjects to detect grooved surfaces pressed gently against the stationary index fingertip of the dominant hand. For the sighted subjects and the blind subjects who did not read Braille, hand dominance was determined by a handedness questionnaire (modified from Oldfield, 1971). For the Braille readers, the dominant hand was defined as the hand preferred for Braille reading. On the basis of these criteria, 26 blind and 45 sighted subjects were tested on their right index fingers and 11 blind and 2 sighted subjects on their left index fingers. The stimulus surfaces were 12.7-mm-diameter Delrin plastic cylindrical rods (Small Parts, Miami Lakes, FL), the ends of which were milled with evenly spaced parallel grooves. Twenty surfaces were used, with groove widths ranging from 0.25 to $3.10 \mathrm{~mm}$ in $0.15-\mathrm{mm}$ increments. Ridge width was equal to groove width. Groove depth was sufficient to prevent the skin from touching the bottom of a groove.

We used a two-interval two-alternative forced choice tracking procedure (see Levitt, 1970) to determine the width of the grooves that each subject could reliably distinguish from a smooth surface of the same size and material (Figure 1). The subject's hand rested palm down on a tabletop through which the stimulus surfaces rose to contact the distal pad of the stationary index finger. Plastic barriers around the finger prevented lateral and forward finger movements; a force sensor placed against the fingernail triggered an error tone in the event of upward, downward, or backward finger motion. A computer-controlled stimulus system (see Goldreich \& Kanics, 2003) pressed the surfaces against the fingertip, with the grooves oriented along the finger's long axis. Stimulus contact velocity was $4 \mathrm{~cm} / \mathrm{sec}$, and contact force was set to either $10 \mathrm{~g}$ or $50 \mathrm{~g}$.

In each trial, a grooved $(\mathrm{G})$ and a smooth (S) surface sequentially contacted the finger (1-sec contact duration, 2-sec intercontact interval), with the presentation order (GS or SG) chosen randomly by the computer program. The subject indicated the perceived stimulus order by pressing one of two response keys with the free hand. Immediate auditory feedback was provided. Groove width started at $1.45 \mathrm{~mm}$, was decremented after two sequential correct responses, and incremented after a single incorrect response. The average of the groove widths at reversal points 4 through 14 indicated the width that the subject was able to detect with $71 \%$ reliability (see Levitt, 1970). This measure, the tactile threshold, is unaffected by starting groove width because the first three reversal points are not included in the average. Each subject completed 10 testing blocks, five at 50 -g contact force alternating with five at 10 -g contact force. The entire testing session, including rest periods, lasted approximately $2 \mathrm{~h}$.

ANOVA and ANCOVA were done with SPSS 6.1 for Macintosh (SPSS Inc., Chicago). We programmed Bayesian statistical tests for multivariate analyses using LabVIEW 5.0 for Macintosh (National Instruments, Austin, TX), as described in detail in Goldreich and Kanics (2003). Briefly, Bayesian parameter estimation using a maineffects linear model (with parameters for group, age, and gender) was used to compare the sighted group with different subgroups of the blind subjects. Posterior probability densities for the group difference parameter were integrated from $0.01 \mathrm{~mm}$ to generate the probabilities that each blind subgroup had enhanced tactile acuity in comparison with the sighted group. Probabilities greater than $90 \%$ were considered statistically significant. Bayesian model selection was used to assess the influence of childhood visual experience, light perception level, and Braille reading on the tactile acuity of the blind subjects. Odds were computed relative to a reference model involving age, gender, and vision (blind or sighted) alone.

\section{RESULTS}

As is shown in Figure 2, and as was confirmed by twoway (force $\times$ block) repeated measures ANOVAs, neither
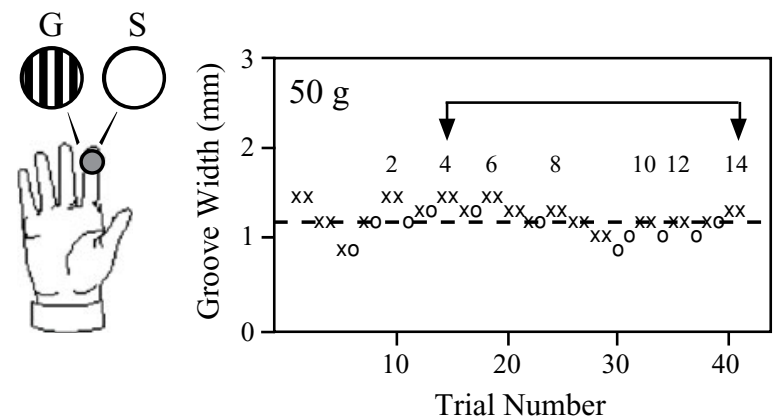

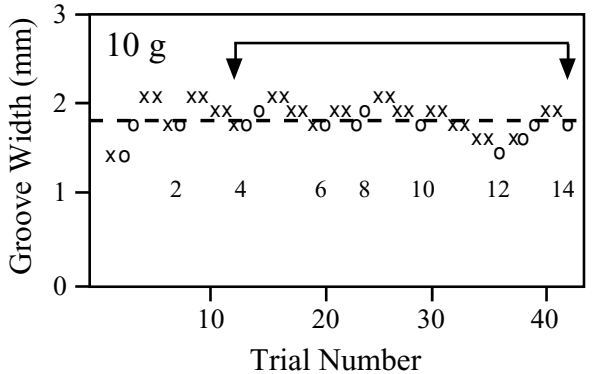

Figure 1. Experimental procedure. During each trial (left), one surface with grooves parallel to the axis of the finger (G) and one smooth surface (S) sequentially contacted the distal pad of the index finger, in random order. Each contact lasted 1 sec; 2 sec elapsed between contacts. The subject indicated the perceived contact order (SG or GS) by pressing a response key with the free hand. Each subject completed 10 testing blocks, five at 50-g contact force alternating with five at $10-\mathrm{g}$ contact force. Shown are the final 50-g (middle) and 10-g (right) testing blocks of a blind woman 60 years 8 months old. Groove width was decreased following two correct $(x)$ responses, and increased following a single incorrect (o) response. Step size was $0.30 \mathrm{~mm}$ until the third reversal of direction in groove width adjustment (in order to converge rapidly onto the subject's approximate threshold) and $0.15 \mathrm{~mm}$ thereafter (to determine the threshold with precision). A block was terminated upon the 14th reversal of direction in groove width adjustment (numbers next to traces). The average of groove widths at reversal points 4 through 14 (bracketed region) was the subject's threshold (dashed lines). 

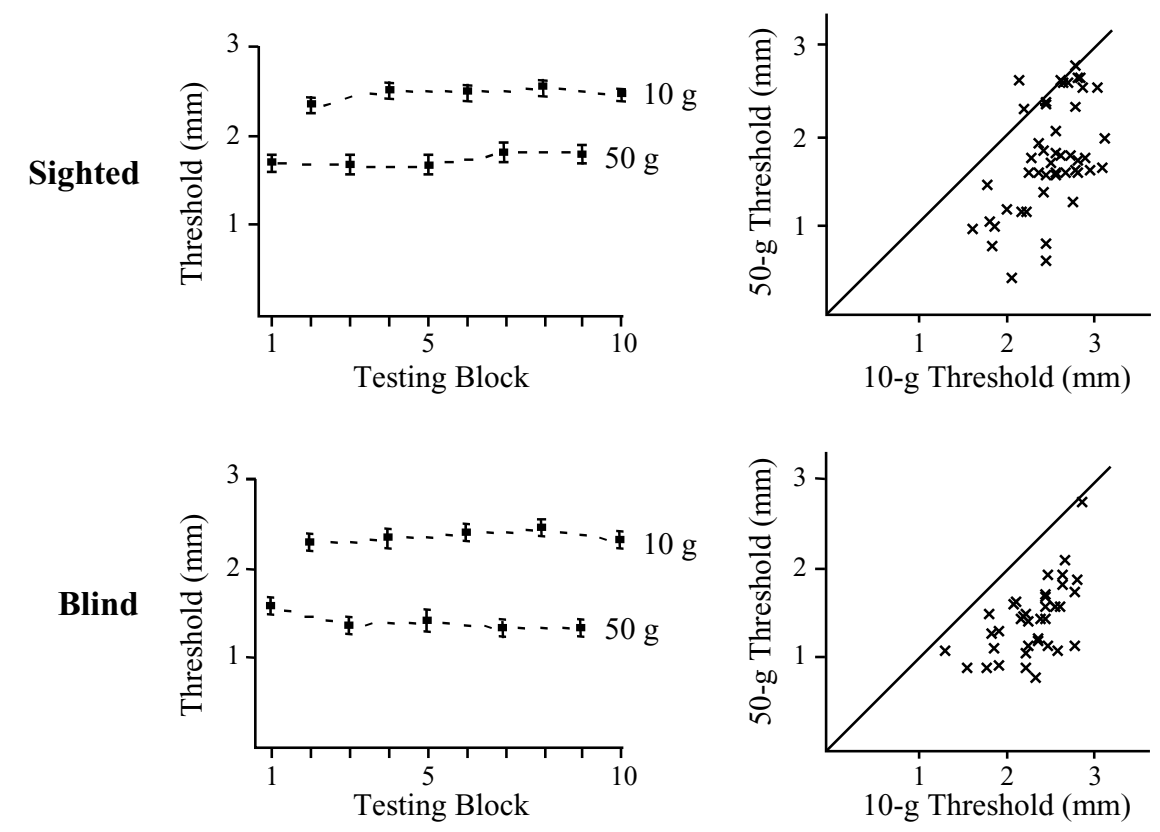

Figure 2. Effects of repeated testing and contact force. Left panels: Group means ( \pm standard errors) of sighted and blind subjects on the five 50-g and five 10-g testing blocks. Right panels: Scatterplots showing effect of contact force. Each point represents the average of a subject's five 10-g thresholds plotted against the average of the subject's five 50-g thresholds. Diagonal lines: $x=y$.

blind nor sighted subjects improved with practice across the 10 testing blocks (blind, $F=0.54, p=.70$; sighted, $F=1.58, p=.18$ ). Contact force, by contrast, exerted a significant effect on tactile acuity in both groups (blind, $F=242.91, p<.001 ;$ sighted, $F=96.32, p<.001)$. The average within-subjects difference between 10 -g and 50 -g thresholds was $0.94 \pm 0.06 \mathrm{~mm}(M \pm S E)$ for blind and $0.74 \pm 0.08 \mathrm{~mm}$ for sighted subjects. No significant force $\times$ block interactions were found. Subjects' average 10- and 50-g thresholds were positively correlated within each group (blind, $r=.57, p<.001$; sighted, $r=$ $.52, p<.001)$, and this correlation was due only partly to the concomitant increase in 10- and 50-g thresholds with subject age (see below). Age-corrected 10- and 50-g thresholds were also significantly correlated (blind, $r=$ $.56, p<.001$; sighted, $r=.46, p<.001)$. Thus, subjects who excelled at the $50 \mathrm{-g}$ force level also excelled at the 10-g level.

To compare the acuity of blind and sighted subjects, we performed an age- and gender-controlled ANCOVA (Figure 3). At both force levels, tactile acuity declined significantly with age (age main effect at contact force of $50 \mathrm{~g}, 0.013 \mathrm{~mm} /$ year, $F=10.4, p<.01$; at contact force of $10 \mathrm{~g}, 0.009 \mathrm{~mm} /$ year, $F=9.3, p<.01$ ) and blind subjects significantly outperformed their sighted peers (vision main effect at contact force of $50 \mathrm{~g}, 0.375 \mathrm{~mm}, F=$ 11.4, $p<.01$; at contact force of $10 \mathrm{~g}, 0.158 \mathrm{~mm}, F=$ $4.4, p<.05)$. At 10 -g contact force, women significantly outperformed men (gender main effect at $10 \mathrm{~g}: 0.182 \mathrm{~mm}$, $F=5.53, p<.05)$. No significant vision $\times$ gender in- teractions were found. No significant differences were observed in the rate at which tactile acuity declined with age in blind and sighted subjects, or between the rates of acuity decline at different force levels.

To summarize the difference in acuity between blind and sighted subjects, we averaged each subject's thresholds across all 10 testing blocks. The average threshold increased with age at a rate of $0.011 \mathrm{~mm} /$ year (with $95 \%$ confidence interval, $0.005-0.017 \mathrm{~mm} /$ year), and blind subjects had on average $0.267 \mathrm{~mm}$ lower thresholds (with $95 \%$ confidence interval, $0.11-0.43 \mathrm{~mm}$ ) than did their sighted peers. Thus, the average blind subject had the tactile acuity of an average sighted subject 24 years younger.

To determine whether the tactile superiority of the blind subjects was limited to Braille readers, to congenitally blind individuals, or to those with no light perception, we divided the blind subjects into those and other subgroups (Figure 4, upper panel). We then used Bayesian parameter estimation to compare each subgroup separately with the sighted group. Each blind subgroup showed greater tactile acuity than did the sighted group. Interestingly, even those blind subjects with normal childhood vision significantly outperformed the sighted subjects, as did those who did not read Braille.

Finally, we searched for any effects of Braille reading, childhood visual experience, and light perception level on the tactile acuity of the blind subjects. A Bayesian model selection analysis confirmed that these factors did not significantly influence tactile acuity, either alone or in any 

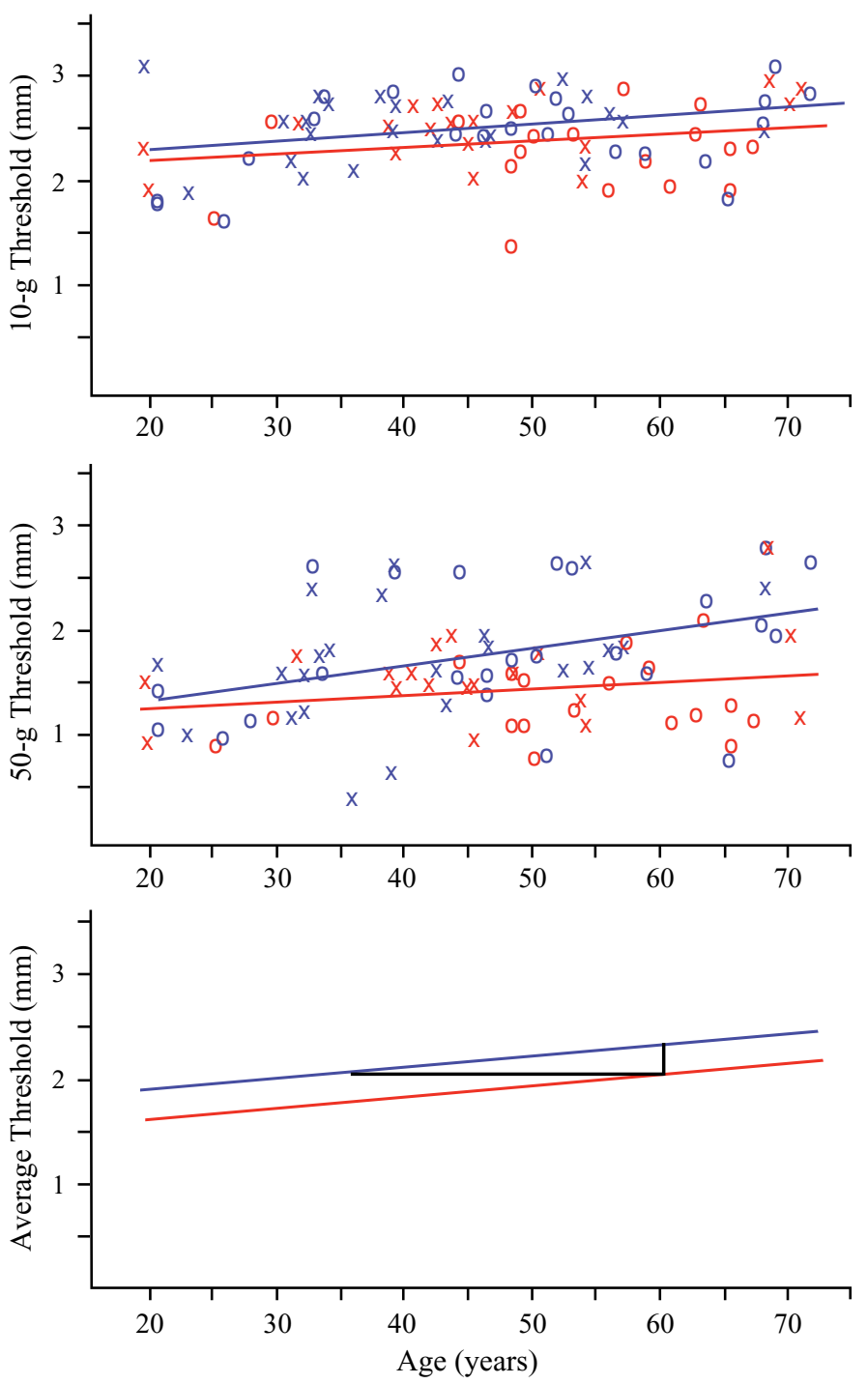

Figure 3. Effects of blindness and age. Each subject's average 10-g (upper panel) and 50-g (middle panel) detection threshold plotted against age. Blind (red), sighted (blue), women (o), men (x). Best-fit linear regression lines are shown for the blind (red) and sighted (blue) groups. Lower panel: Best estimates of blind (red) and sighted (blue) overall average thresholds as function of age. The $0.27-\mathrm{mm}$ lower threshold of blind subjects (vertical line segment) is equivalent to an age difference of 24 years (horizontal line segment).

combination (odds $<1$ for all models). We further categorized Braille readers according to their age at learning Braille, years reading Braille, and daily Braille reading time (Figure 4, lower panel). Again, none of these factors correlated with the tactile acuity of the blind subjects (odds $<1$ for all models).

\section{DISCUSSION}

The data reported here reveal that blind subjects can detect more-finely grooved surfaces than can sighted subjects. Interestingly, tactile acuity among the blind subjects was not influenced significantly by childhood visual deprivation, residual light perception, or Braille reading. As explained below, these findings contribute to a growing literature on the tactile capabilities of blind persons and provide clues to the cause of the blindness-related acuity enhancement.

\section{Passive Tactile Acuity Is Enhanced in Blindness}

Several recent psychophysical studies have provided evidence for the superior passive (finger stationary) tactile spatial abilities of blind persons. Blind subjects have outperformed sighted subjects on grating orientation dis- 

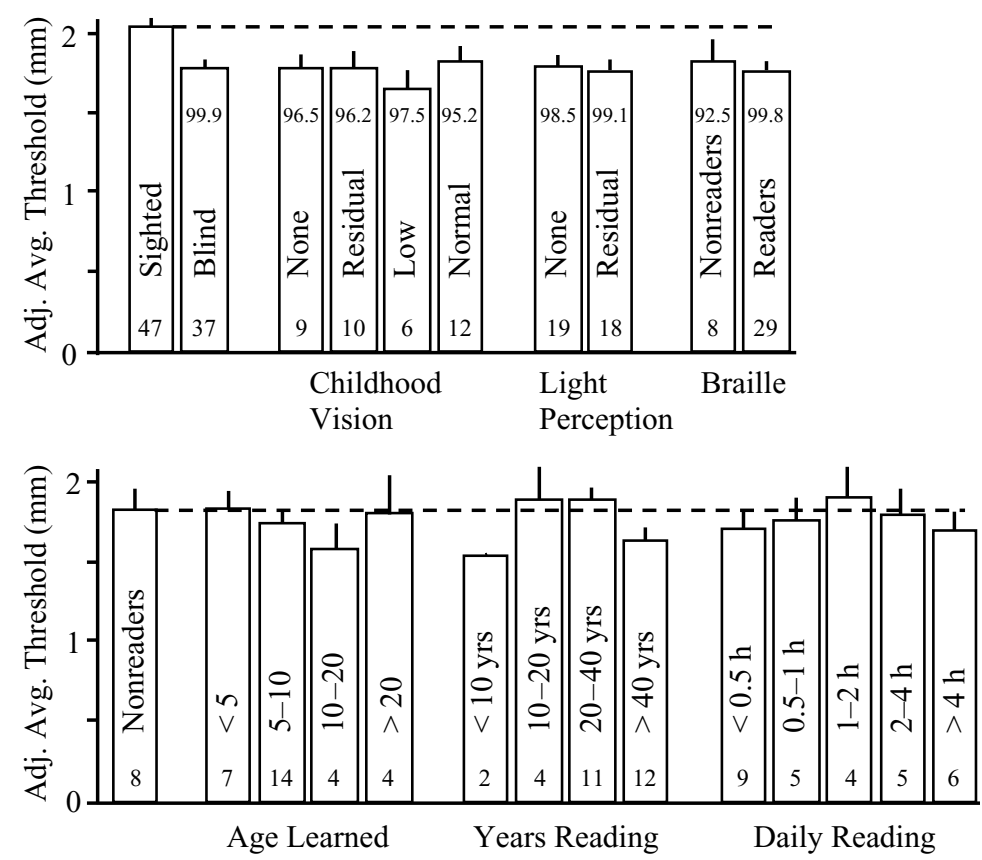

Figure 4. Effects of childhood visual experience, light perception level, and Braille reading. For display purposes only, each subject's average detection threshold was age-adjusted to 45 years old, and thresholds of men were genderadjusted to equivalent female values. Upper panel: Adjusted average detection thresholds of sighted (left; dashed line) and different subgroups of blind subjects. Histogram bars, group means; error bars, standard errors; lower numbers, sample sizes; upper numbers, percentage probability that each blind subgroup has enhanced tactile acuity (compared with sighted). Lower panel: Adjusted average thresholds of blind nonreaders (left; dashed line) and different subgroups of Braille readers.

crimination tasks (see, e.g., Goldreich \& Kanics, 2003; Van Boven et al., 2000; but see also Grant, Thiagarajah, \& Sathian, 2000) and nongrating measures of passive tactile acuity, such as gap detection and line orientation (see Stevens et al., 1996). The present study extends these recent findings by showing superior performance by blind persons on a task involving the detection of tactile gratings. Our study also confirms the dependence of tactile acuity on contact force (see, e.g., Goldreich \& Kanics, 2003) and the well-established decline in tactile spatial acuity with age (see Goldreich \& Kanics, 2003; Stevens et al., 1996; Stevens \& Patterson, 1995; Woodward, 1993), which may result from the loss of SAI afferent axons.

\section{Comparison Between Grating Detection and Grating Orientation Discrimination}

We previously reported that blind subjects outperformed sighted ones on a test of grating orientation discrimination (Goldreich \& Kanics, 2003). In that task, subjects were asked to discriminate between grooves aligned parallel to the finger (i.e., in a proximal-distal orientation) and those aligned transversely. In the present study, by contrast, subjects were required to distinguish grooves aligned parallel to the finger from a completely smooth surface. Thus, in the present study, subjects had to detect (but not necessar- ily to resolve the orientation of) a coarse surface, where we follow Hollins and Risner (2000) in defining coarse surfaces as those with elements (in this case, grooves and ridges) of a dimension greater than 100 microns.

Since the same subjects participated in the two studies, we are able to make a within-subjects comparison of performance on the two tasks (Figure 5). At 50-g contact force, both the blind and the sighted subjects in the present study had thresholds significantly lower than those found previously for grating orientation discrimination (average within-subjects discrimination-minus-detection threshold difference: $0.29 \mathrm{~mm}$, sighted; $0.28 \mathrm{~mm}$, blind). This difference in performance on the two tasks, consistent with the results of Craig (1999) and Gibson and Craig (2002), indicates that both blind and sighted subjects are able to detect a grooved surface without discriminating its orientation.

That it is possible to detect the presence of grooves without perceiving their orientation is not surprising. Grating detection is probably mediated by two distinct neural cues, whereas grating orientation discrimination relies primarily - perhaps exclusively - on just one of these. These two cues (see Johnson \& Phillips, 1981) are the afferent discharge magnitude (e.g., the number of afferent action potentials integrated over the contacted skin area) 
50 Grams

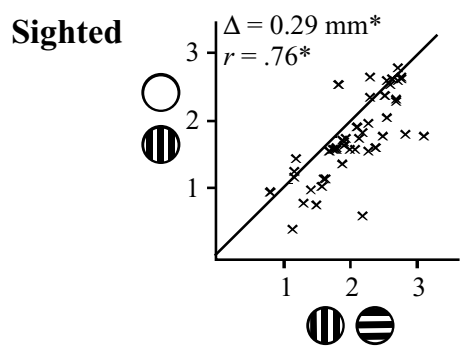

Blind
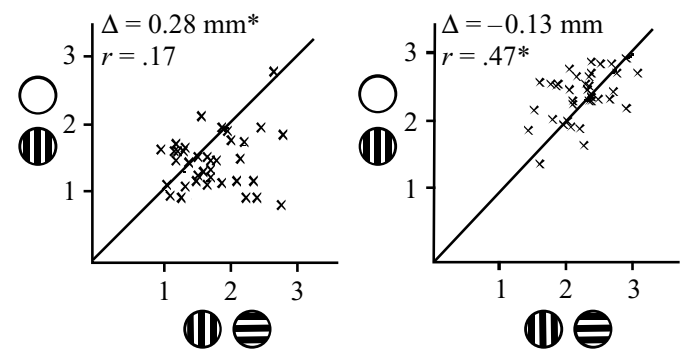

Figure 5. Grating detection versus grating orientation discrimination. Each subject's average grating orientation discrimination threshold ( $x$-axis), obtained in a previous study (Goldreich $\&$ Kanics, 2003), plotted against that subject's average grating detection threshold from the current study ( $y$-axis). Diagonal lines: $x=y \cdot \Delta$ : mean orientation-minus-detection threshold difference. $r$ : correlation coefficient. Axis units: $\mathbf{m m} . \quad$ * $p<.05$.

and the afferent discharge spatial structure (represented by the receptive field locations of activated SAI afferents). At 50-g contact force, the afferent discharge magnitude evoked by a grooved surface probably varies negligibly with groove orientation, provided that the fingerprint ridges in the region of stimulus contact follow roughly circular contours, so that they are never fully aligned with the grooves in the stimulus surface (see Johnson \& Phillips, 1981; Wheat \& Goodwin, 2000). Therefore, to discriminate groove orientation, subjects presumably must attend instead to the spatial structure of the afferent discharge (see Johnson \& Phillips, 1981; Phillips \& Johnson, 1981).

The grating detection task, by contrast, probably provides an afferent discharge magnitude cue in addition to the afferent discharge spatial structure cue. As suggested by Gibson and Craig (2002), the afferent discharge magnitude is likely greater when the finger is contacted by a grating than by a smooth surface, because the edges prevalent in the grating evoke strong responses from the SAI population (Phillips \& Johnson, 1981). In addition, the discharge magnitude of thermoreceptive afferents may aid in grating detection, because the greater skin area contacted by the smooth surface probably results in more efficient transfer of heat from the skin to the contactor. Although all contactors used in our study were at room temperature, 6 of the 84 subjects ( 3 blind and 3 sighted) spontaneously reported perceived temperature differences between the grooved and smooth surfaces. Four subjects reported that the smooth surfaces were cooler, whereas the remaining two were unsure or did not specify. Craig (1999) also re- ports that subjects occasionally commented on temperature differences between smooth and grooved surfaces, although grating detection thresholds did not change significantly when he reduced thermal transfer by warming the contactors to approximately skin temperature.

At 10-g contact force, by contrast, subjects' grating detection and grating orientation discrimination thresholds were equivalent (Figure 5). The reason for subjects' equivalent performance on the two tasks at $10 \mathrm{~g}$ but not at $50 \mathrm{~g}$ is unclear. A plausible explanation is that, at very low contact forces, surfaces with grooves aligned transversely to the finger feel relatively smooth compared with surfaces with grooves aligned parallel to the finger. This situation would effectively convert the grating orientation task at $10 \mathrm{~g}$ into a grating detection task, yielding equivalent performance under the two conditions.

Such a situation might arise because skin curvature is greater transversely than parallel to the long axis of the finger, so that a circular stimulus surface pressed very lightly against the finger might in fact contact a roughly oval region of skin, elongated parallel to the long axis of the finger (the skin, only slightly depressed, would curve away from the stimulator on either side of the finger). Stimulus surfaces with grooves aligned parallel to the finger might then be detected more readily than those aligned transversely, because the groove length in contact with the finger (and therefore afferent discharge magnitude) would in fact be greater in the former situation. As contact force increases from 10 to $50 \mathrm{~g}$, the greater skin indentation would result in a more nearly circular contact profile, and consequently the orientation-related anisotropy in the afferent discharge magnitude would diminish or disappear. In support of this speculation, we note that Essock, Krebs, and Prather (1992), using 16-g contact force, found that subjects were better able to distinguish grooves aligned parallel to the finger than transversely, whereas Craig (1999), using 100-g contact force, found no difference in performance between these two orientations.

We therefore suggest that at 10 -g contact force, grating orientation discrimination and grating detection performance are equivalent, since they both take advantage of the afferent discharge magnitude cue; whereas at 50-g contact force, performance on the two tasks differs, since the former is then based, of necessity, on the afferent discharge's spatial structure, which is more difficult to discern.

In light of the above, the superior performance of the blind subjects compared with the sighted subjects on grating orientation discrimination at $50 \mathrm{~g}$ (see, e.g., Goldreich \& Kanics, 2003) suggests that blind persons more effectively process the spatial information available in the SAI afferent discharge; whereas the blind subjects' superior performance on grating detection and on grating orientation discrimination at $10 \mathrm{~g}$ suggests that they also make more efficient use of afferent discharge magnitude information.

\section{Loss of Sight at Any Age Drives Tactile Acuity Enhancement}

The finding that tactile acuity is enhanced in blindness raises two pressing questions. First, must blindness occur 
early in life for tactile acuity to improve? Second, since blindness is characterized by both loss of sight and increased reliance on nonvisual senses, which of these factors drives the tactile acuity enhancement? Our data indicate that blindness at any age results in enhanced tactile acuity; we find no evidence for a developmental critical period. The data further suggest that the loss of sight itself results in enhanced tactile acuity; enriched tactile experience, if involved in the acuity enhancement, apparently plays a secondary role. The logic behind these conclusions is as follows.

First, the tactile acuity of the blind subjects did not depend upon when they lost vision. Congenitally blind subjects performed equivalently to those with residual, low, or normal childhood vision (Figure 4). Thus, even blind subjects with normal childhood vision significantly outperformed their sighted peers. Similar results were found by Goldreich and Kanics (2003). Additionally, two recent studies indicate that sighted adults deprived of vision for either 5 days (see, e.g., Kauffman, Théoret, \& PascualLeone, 2002) or for just $90 \mathrm{~min}$ (see Facchini \& Aglioti, 2003) show reversible passive tactile acuity gains. Collectively, these results suggest that loss of sight at any age results in enhanced passive tactile acuity and argue against a developmental critical period for the blindness-induced tactile acuity gain. Interestingly, this finding apparently does not generalize to tactile tasks involving temporal order judgments, in which congenitally blind subjects outperform both late-blind and sighted subjects (Röder, Rösler, \& Spence, 2004).

Second, Braille reading experience did not correlate with tactile acuity among the blind subjects. Braille readers, although tested on the preferred reading finger, performed equivalently to blind nonreaders. Furthermore, we found no significant effects on tactile acuity of the age at which Braille was learned, years reading Braille, or daily Braille reading time (Figure 4). Similar results were reported by Goldreich and Kanics (2003). Van Boven et al. (2000) also found no correlation between Braille reading experience and tactile acuity on the reading finger. It therefore appears unlikely that the enhanced tactile acuity of blind persons results primarily from enriched tactile experience.

Nevertheless, the results from several studies suggest that enriched tactile experience may contribute to tactile acuity enhancement. Van Boven et al. (2000) reported that 9 of 13 Braille readers with a preferred reading finger had greater acuity on that finger than on three other fingers tested. Although this result is consistent with the hypothesis that Braille reading improves tactile acuity in the reading finger, a plausible alternative hypothesis is that Braille readers prefer to read with the finger that has the greatest (preexisting) acuity. Stevens et al. (1996) found a correlation between tactile acuity and Braille reading speed among blind subjects. This result could indicate either that more-experienced readers (who tend to read fastest) have acquired greater tactile acuity; or, alternatively, that greater acuity enables more rapid reading. Finally, Ragert, Schmidt, Altenmüller, and Dinse (2004) report that sighted pianists have significantly lower two-point thresholds than do control subjects, and that their twopoint thresholds correlate inversely with daily piano practice time. Although the validity of the two-point threshold as a measure of tactile acuity has been questioned (see, e.g., Craig \& Johnson, 2000), this result suggests that tactile experience contributes to perceptual enhancement.

\section{Crossmodal Plasticity May Mediate the Tactile Acuity Enhancement}

What neural mechanism underlies the tactile superiority of blind persons? Adaptive changes in cerebrocortical maps-specifically, somatosensory cortical plasticity and crossmodal plasticity-have been suggested as possible mechanisms (for a review, see Rauschecker, 2002). Somatosensory cortical plasticity describes the capacity of the sensory homunculus to change as a result of tactile experience. For example, repeated use of a fingertip for Braille reading enlarges that fingertip's representation within the homunculus (see, e.g., Pascual-Leone \& Torres, 1993; Sterr, Müller, Elbert, Rockstroh, \& Taub, 1999); the representations of nonreading fingers apparently remain unchanged (see Pascual-Leone \& Torres, 1993). As discussed above, however, blind nonreaders as well as Braille readers significantly outperformed sighted subjects. Indeed, Braille reading did not correlate with tactile acuity among blind subjects. Somatosensory cortical plasticity, then, whatever its functional consequence might be, seems an unlikely explanation for the superior tactile acuity of blind persons.

Crossmodal plasticity, on the other hand, is a promising candidate. Like the tactile acuity enhancement reported in the present study, this recruitment of normally visual cortical areas for tactile processing in blind persons is apparently triggered by the loss of sight itself (see, e.g., Sadato, Okada, Kubota, \& Yonekura, 2004). Many of the same occipital cortical areas that in sighted persons subserve vision activate when blind persons perform tactile discrimination tasks (see Gizewski et al., 2003), presumably because modulatory tactile inputs to these occipital areas (see Amedi, Malach, Hendler, Peled, \& Zohary, 2001; Macaluso, Frith, \& Driver, 2002) strengthen upon loss of competing visual inputs (see Wittenberg, Werhahn, Wassermann, Herscovitch, \& Cohen, 2004). Blind persons may have enhanced tactile acuity because they are able to utilize these normally visual areas, in addition to the conventional somatosensory areas, for tactile processing (Cohen et al., 1997).

Crossmodal plasticity, unlike somatosensory cortical plasticity, occurs in blind subjects who have not learned to read Braille (see, e.g., Sadato et al., 2004) as well as in Braille readers (see, e.g., Burton et al., 2002; Gizewski et al., 2003; Sadato et al., 1998). Furthermore, crossmodal plasticity occurs not only in early blind persons (see, e.g., Gizewski et al., 2003; Sadato et al., 1998), but alsoless extensively, according to several studies (Cohen et al., 1999; Sadato, Okada, Honda, \& Yonekura, 2002; Wittenberg et al., 2004) - in those who became blind as adults (see, e.g., Büchel, Price, Frackowiak, \& Friston, 
1998; Burton et al., 2002; Sadato et al., 2004), and even in sighted adults blindfolded for 5 consecutive days (see Pascual-Leone \& Hamilton, 2001). Finally, crossmodal plasticity occurs in blind persons with residual light perception (see Sadato et al., 2004) as well as in those without light perception (see Gizewski et al., 2003). Thus, crossmodal plasticity has been detected in each subgroup of blind persons shown in the present study to have enhanced tactile acuity.

If crossmodal plasticity provides the neural basis for the enhancement of tactile acuity in blindness, then it seems reasonable to hypothesize that related mechanisms underlie other forms of sensory compensation, such as the enhanced auditory capabilities of blind persons (see, e.g., Hugdahl et al., 2004; Lessard et al., 1998; Röder et al., 1999) and the altered tactile and visual perceptual abilities of deaf persons (see Levänen \& Hamdorf, 2001; Proksch $\&$ Bavelier, 2002). Consistent with this hypothesis, normally visual cortical areas in blind persons acquire not only tactile, but also auditory responsiveness (see, e.g., Gougoux et al., 2005; Weeks et al., 2000), whereas normally auditory cortical regions in deaf persons are activated by vision (see Finney et al., 2003) and by touch (see Levänen et al., 1998).

\section{REFERENCES}

Amedi, A., Malach, R., Hendler, T., Peled, S., \& Zohary, E. (2001) Visuo-haptic object-related activation in the ventral visual pathway. Nature Neuroscience, 4, 324-330.

Axelrod, S. (1959). Effects of early blindness: Performance of blind and sighted children on tactile and auditory tasks. New York: American Foundation for the Blind.

Büchel, C., Price, C., Frackowiak, R. S. J., \& Friston, K. (1998). Different activation patterns in the visual cortex of late and congenitally blind subjects. Brain, 121, 409-419.

Burton, H., Snyder, A. Z., Conturo, T. E., Akbudak, E., Ollinger, J. M., \& RaIChle, M. E. (2002). Adaptive changes in early and late blind: A fMRI study of Braille reading. Journal of Neurophysiology, 87, 589-607.

Cohen, L. G., Celnik, P., Pascual-Leone, A., Corwell, B., Faiz, L., Dambrosia, J., ET AL. (1997). Functional relevance of cross-modal plasticity in blind humans. Nature, 389, 180-183.

Cohen, L. G., Weeks, R. A., Sadato, N., Celnik, P., Ishit, K., \& Hallett, M. (1999). Period of susceptibility for cross-modal plasticity in the blind. Annals of Neurology, 45, 451-460.

Craig, J. C. (1999). Grating orientation as a measure of tactile spatial acuity. Somatosensory \& Motor Research, 16, 197-206.

Craig, J. C., \& Johnson, K. O. (2000). The two-point threshold: Not a measure of tactile spatial resolution. Current Directions in Psychological Science, 9, 29-32.

Essock, E. A., Krebs, W. K., \& Prather, J. R. (1992). An anisotropy of human tactile sensitivity and its relation to the visual oblique effect. Experimental Brain Research, 91, 520-524.

Facchini, S., \& Aglioti, S. M. (2003). Short term light deprivation increases tactile spatial acuity in humans. Neurology, 60, 1998-1999.

Finney, E. M., Clementz, B. A., HickoK, G., \& Dobkins, K. R. (2003). Visual stimuli activate auditory cortex in deaf subjects: Evidence from MEG. NeuroReport, 14, 1425-1427.

Gibson, G. O., \& Craig, J. C. (2002). Relative roles of spatial and intensive cues in the discrimination of spatial tactile stimuli. Perception \& Psychophysics, 64, 1095-1107.

Gizewski, E. R., Gasser, T., DE Greiff, A., Boehm, A., \& Forsting, M. (2003). Cross-modal plasticity for sensory and motor actitvation patterns in blind subjects. NeuroImage, 19, 968-975.
Goldreich, D., \& Kanics, I. M. (2003). Tactile acuity is enhanced in blindness. Journal of Neuroscience, 23, 3439-3445.

Gougoux, F., Zatorre, R. J., Lassonde, M., Voss, P., \& Lepore, F. (2005). A functional neuroimaging study of sound localization: Visual cortex activity predicts performance in early-blind individuals. PLoS Biology, 3, e27.

Grant, A. C., Thiagarajah, M. C., \& Sathian, K. (2000). Tactile perception in blind Braille readers: A psychophysical study of acuity and hyperacuity using gratings and dot patterns. Perception \& Psychophysics, 62, 301-312.

Hollins, M. (1989). Understanding blindness: An integrative approach. Hillsdale, NJ: Erlbaum.

Hollins, M., \& Risner, S. R. (2000). Evidence for the duplex theory of tactile texture perception. Perception \& Psychophysics, 62, 695-705.

Hugdahl, K., EK, M., Takio, F., Rintee, T., Tuomainen, J., HaArala, C., \& HÄMÄLÄINEN, H. (2004). Blind individuals show enhanced perceptual and attentional sensitivity for identification of speech sounds. Cognitive Brain Research, 19, 28-32.

Johnson, K. O., \& PhILliPs, J. R. (1981). Tactile spatial resolution: I. Two-point discrimination, gap detection, grating resolution, and letter recognition. Journal of Neurophysiology, 46, 1177-1192.

Kauffman, T., Théoret, H., \& Pascual-Leone, A. (2002). Braille character discrimination in blindfolded human subjects. NeuroReport, 13, 571-574.

Lessard, N., PAré, M., Lepore, F., \& Lassonde, M. (1998). Early-blind human subjects localize sound sources better than sighted subjects. Nature, 395, 278-280.

LEVÄNEN, S., \& HAMdorf, D. (2001). Feeling vibrations: Enhanced tactile sensitivity in congenitally deaf humans. Neuroscience Letters, 301, 75-77.

LEVÄNEN, S., Jousmaki, V., \& HARI, R. (1998). Vibration-induced auditory-cortex activation in a congenitally deaf adult. Current Biology, 8, 869-872.

LEviTT, H. (1970). Transformed up-down methods in psychoacoustics. Journal of the Acoustical Society of America, 49, 467-477.

Macaluso, E., Frith, C. D., \& Driver, J. (2002). Crossmodal spatial influences of touch on extrastriate visual areas take current gaze direction into account. Neuron, 34, 647-658.

OLDFIELD, R. C. (1971). The assessment and analysis of handedness: The Edinburgh inventory. Neuropsychologia, 9, 97-113.

Pascual-Leone, A., \& Hamilton, R. (2001). The metamodal organization of the brain. Progress in Brain Research, 134, 427-445.

Pascual-Leone, A., \& Torres, F. (1993). Plasticity of the sensorimotor cortex representation of the reading finger in Braille readers. Brain, 116, 39-52.

Phillips, J. R., \& Johnson, K. O. (1981). Tactile spatial resolution: II. Neural representation of bars, edges, and gratings in monkey primary afferents. Journal of Neurophysiology, 46, 1192-1203.

Proksch, J., \& Bavelier, D. (2002). Changes in the spatial distribution of visual attention after early deafness. Journal of Cognitive Neuroscience, 14, 687-701.

Ragert, P., Schmidt, A., Altenmüller, E., \& Dinse, H. R. (2004). Superior tactile performance and learning in professional pianists: Evidence for meta-plasticity in musicians. European Journal of Neuroscience, 19, 473-478.

RAuscheCKer, J. P. (2002). Cortical map plasticity in animals and humans. Progress in Brain Research, 138, 73-88.

RöDER, B., RöSLER, F., \& SPENCE, C. (2004). Early vision impairs tactile perception in the blind. Current Biology, 14, 121-124.

Röder, B., Teder-Sälejärvi, W., Sterr, A., Rösler, F., Hillyard, S. A., \& Neville, H. J. (1999). Improved auditory spatial tuning in blind humans. Nature, 400, 162-166.

Sadato, N., OKada, T., Honda, M., \& YoneKura, Y. (2002). Critical period for cross-modal plasticity in blind humans: A functional MRI study. NeuroImage, 16, 389-400.

Sadato, N., Okada, T., Kubota, K., \& Yonekura, Y. (2004). Tactile discrimination activates the visual cortex of the recently blind naive to Braille: A functional magnetic resonance imaging study in humans. Neuroscience Letters, 359, 49-52.

Sadato, N., Pascual-Leone, A., Grafman, J., Deiber, M. P., 
IвAÑEZ, V., \& HaLLETt, M. (1998). Neural networks for Braille reading by the blind. Brain, 121, 1213-1229.

Sterr, A., Müller, M., Elbert, T., Rockstroh, B., \& Taub, E. (1999). Development of cortical reorganization in the somatosensory cortex of adult Braille students. Electroencephalography \& Clinical Neurophysiology, 49(Suppl.), 292-298.

Stevens, J. C., Foulke, E., \& Patterson, M. Q. (1996). Tactile acuity, aging, and Braille reading in long-term blindness. Journal of Experimental Psychology: Applied, 2, 91-106.

Stevens, J. C., \& Patterson, M. Q. (1995). Dimensions of spatial acuity in the touch sense: Changes over the life span. Somatosensory \& Motor Research, 12, 29-47.

Van Boven, R. W., Hamilton, R. H., Kauffman, T., Keenan, J. P., \& Pascual-Leone, A. (2000). Tactile spatial resolution in blind Braille readers. Neurology, 54, 2230-2236.

Warren, D. H. (1978). Perception by the blind. In E. C. Carterette \& M. P. Friedman (Eds.), Perceptual ecology (Handbook of Perception, Vol. 10, pp. 65-90). New York: Academic Press.
Weeks, R., Horwitz, B., Aziz-Sultan, A., Tian, B., Wessinger, C. M., CoHEN, L. G., ET AL. (2000). A positron emission tomographic study of auditory localization in the congenitally blind. Journal of Neuroscience, 20, 2664-2672.

WheAt, H. E., \& Goodwin, A. W. (2000). Tactile discrimination of gaps by slowly adapting afferents: Effects of population parameters and anisotropy in the fingerpad. Journal of Neurophysiology, 84, 14301444 .

Wittenberg, G. F., Werhahn, K. J., Wassermann, E. M., HerscoVITCH, P., \& CohEN, L. G. (2004). Functional connectivity between somatosensory and visual cortex in early blind humans. European Journal of Neuroscience, 20, 1923-1927.

WoODWARD, K. L. (1993). The relationship between skin compliance, age, gender, and tactile discriminative thresholds in humans. Somatosensory \& Motor Research, 10, 63-67.

(Manuscript received December 8, 2004; revision accepted for publication December 16, 2005.) 